\title{
Stenting the ureteroneocystostomy reduces urological complications in kidney transplantation: a noninferiority randomized controlled trial, SPLINT trial
}

Liselotte S. S. Ooms ${ }^{1}$ (D), Robert C. Minnee ${ }^{1}$, Frank J. M. F. Dor ${ }^{1}$, Diederik J. A. N. Kimenai ${ }^{1}$, Khe C. K. Tran ${ }^{1}$, Hermien Hartog ${ }^{1}$, Jacqueline van de Wetering ${ }^{2}$, Sten P. Willemsen ${ }^{3}$, Jan N. M. IJzermans ${ }^{1}$ \& Turkan Terkivatan ${ }^{1}$

1 Department of Surgery, Erasmus MC, University Medical Center, Rotterdam, The Netherlands

2 Department of Nephrology, Erasmus MC, University Medical Center, Rotterdam, The Netherlands

3 Department of Biostatistics, Erasmus MC, University Medical Center, Rotterdam, The Netherlands

\section{Correspondence}

Dr. Turkan Terkivatan, Department of Surgery, Erasmus MC, University Medical Center, PO BOX 20403000

CA Rotterdam, the Netherlands.

Tel.: +31 107040 704;

e-mail: t.terkivatan@erasmusmc.nl

The trial was registered at the (Dutch) Netherlands Trial Registry: Trial NL4358 (NTR4498).

\begin{abstract}
SUMMIARY
The role of ureteral stents in living-donor kidney transplantation remains uncertain. In this randomized controlled trial (SPLINT), we compared urological complications in living-donor kidney transplantations performed with or without stents. We included 200 consecutive patients that received living-donor kidney transplantations at the Erasmus MC, University Medical Center, Rotterdam. Patients (124 males, 76 females, mean age $54 \pm 13$ ) were randomized for suprapubic externalized single $J$ stents $(N=100)$ or no stent $(N=100)$. The primary outcome was the probability of a percutaneous nephrostomy insertion (PCN) during a 12-month follow-up. To assess whether no stenting is noninferior to stenting, we allowed the probability of a PCN to increase by at most 5\% (this is the noninferiority margin). Baseline characteristics were comparable between groups. In the no-stent group, there were more PCN insertions, $14 \%$ (95\% CI $4.3-$ $23.7 \%$ ); urinary leakages, $12 \%$ (95\% CI 5.4-21.3\%); and surgical re-interventions because of urological complications, $8 \%$ (95\% CI $1.5-14.5 \%$ ). The stent group had more hematuria, 26\% (95\% CI 13.1-38.9\%); and graft rejections, $15 \%$ (95\% CI 2.7-27.3\%). Patients in both groups had similar mean GFRs at several time points. Besides a better Euro-Qol-5D in the no-stent group at 2 and 6 weeks postoperative, similar quality of life was reported based on SF-36 and Euro-Qol-5D scores. In this trial, noninferiority has not been demonstrated for no-stent placement in relation to the number urological complications.
\end{abstract}

Transplant International 2020;

Key words

kidney, stent, surgery, transplantation

Received: 31 October 2019; Revision requested: 6 December 2019; Accepted: 4 May 2020

\section{Introduction}

Kidney transplantation is the optimal treatment offering long-term benefits to the majority of patients with chronic kidney failure. However, urological complications after kidney transplantation, such as urinary leakage and ureteral strictures, are associated with significant morbidity, surgical and radiological 
interventions, prolonged hospital stays, and even mortality. Most urological complications are related to the ureteroneocystostomy, and they are treated with a percutaneous nephrostomy (PCN) $[1,2]$.

The role of ureteral stents in living-donor kidney transplantations remains uncertain. A Cochrane review, published in October 2005 and revised in 2013, suggests that routine prophylactic stenting reduces the incidence of major urological complications. However, there are some limitations to this Cochrane review. First of all, most included studies are from 1995 to 2000. As in the last 20 years many improvements have been made in the immunosuppressive treatment, we wonder how representative these data are for current medicine. Secondarily, different kinds of stents (lengths and caliber) have been used and none of them include an externalized stent. Furthermore, the study designs of the seven included articles were heterogeneous with different types of donors, intervention periods, outcome assessments, and statistical analysis [3,4]. Stent placement also has some disadvantages. The complications associated with stents include infections, obstructions, stent migrations, breakage, stone formation, hematuria, and secondary ureteral obstructions [4-9].

In this trial (Stent PLacement IN living-donor kidney Transplantation, SPLINT), we tested the hypothesis that omitting a ureteral stent in kidney transplantation might be as effective as stenting, and it might even reduce the number of urological complications, because of the absence of stent-related problems. We also followed patients for 1 year to evaluate quality of life (QOL).

\section{Methods}

\section{Study design}

In this randomized controlled trial, we included all patients that received a living-donor kidney transplantation at the Erasmus MC, University Medical Center, Rotterdam, the Netherlands, between April 2014 and March 2017. Exclusion criteria were as follows: declined informed consent, age $<18$ years, a reconstructed urinary tract or conduit after total or partial cystectomy, bladder dysfunction that required continuous or intermittent catheterization, and a donor kidney with duplicated ureters. Patients with primary focal segmental glomerulosclerosis (FSGS) that still had residual urinary output were also excluded. FSGS is known to recur rapidly in kidney grafts, in which case the first sign is proteinuria. An externalized stent allows one to distinguish whether proteinuria originated in the transplanted kidney or the native kidneys. Furthermore, we excluded recipients that were included in another ongoing clinical trial.

We randomized 200 patients to either stent placement $\left(N=100\right.$, Teleflex ${ }^{\circledR}$, suprapubic externalized single J stent, $7 \mathrm{fr})$ or no-stent placement $(N=100)$. In our center, the external stent has been standard care for several years. Randomization was performed with a concealed opaque envelope system prepared by an independent statistician at the Erasmus MC, University Medical Center, Rotterdam. Patients were randomized after intubation in the operating room. As a result of the use of an externalized stent, blinding was not possible. There were no blocks and no stratification methods used during randomization.

The Medical Ethics Committee of the Erasmus MC, University Medical Center, Rotterdam, approved the trial protocol (MEC-2013-196), and the study was registered at the (Dutch) Netherlands Trial Registry: Trial NL4358 (NTR4498).

\section{Surgical technique}

The donor nephrectomy was performed with either a fully laparoscopic, a robot-assisted, or hand-assisted retroperitoneoscopic approach. The kidney recipients underwent transplantation with an extraperitoneal approach to the iliac fossa. Firstly, the renal vein was anastomosed to the external iliac vein, followed by the renal artery that was anastomosed to the external iliac artery. Then, an extravesical ureteroneocystostomy was performed, as described by Lich-Gregoir [10,11]. The detrusor muscle was closed over the anastomosis with one or two interrupted absorbable sutures to create a submucosal tunnel, with an antireflux mechanism. The stent group received a 7 -fr suprapubic externalized single $J$ stent $\left(\right.$ Teleflex ${ }^{\circledR}$ ), and it was removed 9 days postoperatively. A transurethral urinary bladder catheter was placed according to standard care in all patients; this catheter was removed after 7 days. All patient had a nuclear renogram scan and an ultrasound one day after surgery. Furthermore, daily serum creatinine levels were determined during hospital stay.

\section{Power calculation}

The SPLINT trial was designed as a noninferiority study. It was powered to demonstrate that omitting a stent would not lead to a relevant increase in the 
urological complication rate, that is, the percentage of required PCN drainages. To show that the increase in patients without a stent requiring a $\mathrm{PCN}$ is at most $5 \%$ (noninferiority margin), 96 patients per arm were required (one-side alpha $=0.025$, power $=90 \%$ ). This calculation was based on the assumption that among patients that received stents, $20 \%$ would require a $\mathrm{PCN}$ [1], and among patients that received no stent, 9\% would require a PCN [2]. To allow room for a few nonevaluable cases, we randomized 100 patients per arm.

\section{Definitions}

Baseline data of the recipients included gender, age, American Society of Anesthesiologists (ASA) classification, number of previous transplantations, body mass index (BMI), warm and cold ischemia times, and preemptive transplantations (prior to starting dialysis). Our primary outcome was a PCN insertion within 12 months. Indications for a PCN insertion were as follows: urinary leakage (detected with a nuclear renogram scan or demonstrated by high creatinine levels in the fluid excretion from the wound or from the drain) or a rise in serum creatinine combined with hydronephrosis (detected with ultrasound). Our secondary outcome was graft function, based on the glomerular filtration rate (GFR), duration of surgery, perioperative blood loss, any surgical re-intervention performed within $<12$ months of kidney transplantation (including nonurological re-interventions), length of hospital stay, hematuria (defined as macroscopic hematuria during hospital admission), urinary tract infection (UTI), and graft rejection $<1$ month after kidney transplantation. UTI was scored in case of a urinary culture with a bacterial load of $\geq 10^{5} \mathrm{CFU} / \mathrm{ml}$ that was treated with antibiotics. Rejection was scored if patients received antirejection treatment (methylprednisolone intravenous, IVIG, alemtuzumab, r-ATG). History of smoking included current or past smokers.

\section{Quality of life questionnaires}

We evaluated QOL, health state, work effort, and disabilities in daily life with two validated questionnaires: the Euro-Qol-5D and the Short Form survey 36 (SF-36) $[12,13]$. All questionnaires were completed preoperatively and at different time points postoperatively (at 2 and 6 weeks and at 3, 6, 9, and 12 months). Repeated QOL measurements were compared with a mixedeffects model for repeated measurements.

\section{Immunosuppressive treatment}

Immunosuppressive treatments included intravenous basiliximab as induction therapy, given on the day of surgery and on day 4 post-transplantation. Postoperative immunosuppression also included tacrolimus, mycophenolate mofetil, and prednisone. The prednisone was tapered off over time and discontinued at 4 months after transplantation.

\section{Statistical analysis}

All analyses were performed with IBM SPSS Statistics for Windows (version 21.0. Armonk, NY, USA: IBM Corp) and $R 3.5$ [R Core Team (2012); a language and environment for statistical computing. $\mathrm{R}$ Foundation for Statistical Computing, Vienna, Austria. ISBN 3900051-07-0, URL http://www.R-project.org/]. We performed an intention-to-treat analysis. Therefore, patients were analyzed in the group in which they were originally allocated. For the primary parameter, we calculated beta with a $95 \%$ confidence interval $(95 \%$ CI $)$ using a generalized linear model for the risk difference for binominal variable and univariate analysis of variance for continuous variable. As the number of urinary leakage was 0 , we were not able to use the generalized linear model, here, we calculated an exact confidence interval for a risk difference [14].

For secondary parameters: Continuous variables with a distribution that is approximately normal are presented as the mean and standard deviation, and comparisons were evaluated with the independent $t$-test. Variables with skewed distributions are presented as the median (range), and comparisons were evaluated with the Mann-Whitney $U$-test. Categorical variables are presented as numbers with percentages, and comparisons were evaluated with the chi-square test.

\section{Results}

\section{Baseline characteristics}

Between April 2014 and March 2017, 200 patients were included in the SPLINT trial. Because of perioperative difficulties, one patient received a stent, although he was allocated to the no-stent group. As this is an intention-to-treat analyses, this patient was analyzed in the no-stent group. The cohort comprised 124 males and 76 females with a mean age of $54 \pm 13$ years. Baseline characteristics were comparable for both groups (Table 1). 
Table 1. Baseline characteristics

\begin{tabular}{|c|c|c|c|c|}
\hline Characteristic & Total $(N=200)$ & No stent $(N=100)$ & Stent $(N=100)$ & $P$-value \\
\hline \multicolumn{5}{|l|}{ Recipient, gender N (\%) } \\
\hline$M$ & $124(62)$ & $63(63)$ & $61(61)$ & \multirow[t]{2}{*}{0.771} \\
\hline $\mathrm{F}$ & $76(38)$ & $37(37)$ & $39(39)$ & \\
\hline Recipient age, years; mean \pm SD & $54 \pm 13$ & $55 \pm 13$ & $52 \pm 14$ & 0.170 \\
\hline \multicolumn{5}{|l|}{ Donor gender, $N(\%)$} \\
\hline $\mathrm{M}$ & $81(41)$ & $45(45)$ & $36(36)$ & \multirow[t]{2}{*}{0.195} \\
\hline $\mathrm{F}$ & $119(59)$ & $55(55)$ & $64(64)$ & \\
\hline Donor age, years; mean \pm SD & $54 \pm 12$ & $54 \pm 12$ & $53 \pm 13$ & 0.572 \\
\hline \multicolumn{5}{|l|}{ ASA, $N(\%)$} \\
\hline 2 & $28(14)$ & $12(12)$ & $16(16)$ & \multirow[t]{3}{*}{0.524} \\
\hline 3 & $164(82)$ & $85(85)$ & $79(79)$ & \\
\hline 4 & $8(4)$ & $3(3)$ & $5(5)$ & \\
\hline \multicolumn{5}{|l|}{ Number of KTs, $N(\%)$} \\
\hline 1 & $173(86)$ & $90(90)$ & $83(83)$ & \multirow[t]{3}{*}{0.239} \\
\hline 2 & $19(10)$ & $6(6)$ & $13(13)$ & \\
\hline 3 & $8(4)$ & $4(4)$ & $4(4)$ & \\
\hline Laparoscopic technique, N (\%) & $123(62)$ & $61(61)$ & $62(62)$ & 0.884 \\
\hline Recipient BMI, mean \pm SD & $27 \pm 5$ & $28 \pm 5$ & $27 \pm 5$ & 0.408 \\
\hline History of smoking, $N(\%)$ & $123(61)$ & $59(59)$ & $64(64)$ & 0.467 \\
\hline Pre-emptive KT, N (\%) & $102(51)$ & $48(48)$ & $54(54)$ & 0.396 \\
\hline Residual urinary production, $N(\%)$ & $172(86)$ & $83(83)$ & $89(89)$ & 0.221 \\
\hline Ureteral length, $\mathrm{cm}$; mean $\pm \mathrm{SD}$ & $9 \pm 2$ & $9 \pm 2$ & $9 \pm 2$ & 0.367 \\
\hline First warm ischemia time, min; mean $\pm S D$ & $3 \pm 1$ & $3 \pm 1$ & $3 \pm 2$ & 0.196 \\
\hline Cold ischemia time, min; mean $\pm S D$ & $140 \pm 29$ & $142 \pm 31$ & $139 \pm 27$ & 0.494 \\
\hline Second warm ischemia time, min; mean \pm SD & $20 \pm 7$ & $20 \pm 7$ & $20 \pm 8$ & 0.876 \\
\hline
\end{tabular}

ASA, American Society of Anesthesiologists; BMI, body mass index; F, female; KT, kidney transplantation; M, male; $N$, number; SD, standard deviation.

\section{Urological complications}

In the no-stent group, 22 patients (22\%) received a PCN after transplantation (Table 2). Of this group, 11 patients received a PCN because of urinary leakage. Five urinary leakages were resolved without further interventions; four patients required a surgical re-intervention to treat the urinary leakage; and two patients underwent both an antegrade balloon dilatation and a surgical reintervention. One patient in the no-stent group with urinary leakage did not receive a PCN, but underwent immediate surgical repair. In the no-stent group, another 11 patients received a PCN because of hydronephrosis. Of these patients, eight did not require an additional intervention, one patient underwent a balloon dilatation, and two patients received both a balloon dilatation and a surgical re-intervention. One patient in the no-stent group with hydronephrosis because of a blood clot in the ureter did not receive a $\mathrm{PCN}$, but underwent surgical repair directly.

In the stent group, eight patients $(8 \%)$ received a PCN after transplantation, all because of hydronephrosis. One patient underwent balloon dilatation, and two patients underwent a balloon dilatation, followed by a surgical re-intervention.

To assess whether no stenting is noninferior to stenting, we allowed an increase of at most $5 \%$ in the number of patients without stent requiring a PCN (i.e., a noninferiority margin of 5\%). There were more PCN insertions in the no-stent group, $14 \%$ (95\% CI $4.3-$ $23.7 \%$ ). Moreover, compared to the stent group, the no-stent group had more urinary leakages, 12\% (95\% CI 5.4-21.3\%); and surgical re-interventions because of urological complications, $8 \%$ (95\% CI $1.5-14.5 \%$ ). As the CI extends below the noninferiority margin, noninferiority of no-stent placement had not been demonstrated. The number of PCNs placed because of hydronephrosis was similar between groups. Details are shown in Fig. 1 and Table 2.

\section{Overall outcome}

There were no significant differences between the stent and no-stent group regarding the duration of surgery, 
Table 2. Urological complications within 12 months

\begin{tabular}{|c|c|c|c|c|c|}
\hline Characteristics & $\begin{array}{l}\text { Total } \\
(N=200)\end{array}$ & $\begin{array}{l}\text { No stent } \\
(N=100)\end{array}$ & $\begin{array}{l}\text { Stent } \\
(N=100)\end{array}$ & $\begin{array}{l}\text { Risk } \\
\text { difference (\%) }\end{array}$ & $95 \% \mathrm{Cl}$ \\
\hline PCN insertion, $N(\%)$ & $30(15)$ & $22(22)$ & $8(8)$ & 14 & 4.3 to 23.7 \\
\hline Urinary leakage, N (\%) & $12(6)$ & $12(12)$ & $0(0)$ & 12 & 5.4 to 21.3 \\
\hline Hydronephrosis, N (\%) & $20(10)$ & $12(12)$ & $8(8)$ & 4 & -4.3 to 12.3 \\
\hline $\begin{array}{l}\text { Surgical re-intervention because of } \\
\text { urological complications, } N(\%)\end{array}$ & $12(6)$ & $10(10)$ & $2(2)$ & 8 & 1.5 to 14.5 \\
\hline Antegrade balloon dilatation, $N(\%)$ & $8(4)$ & $5(5)$ & $3(3)$ & 2 & -3.4 to 7.4 \\
\hline
\end{tabular}

$\mathrm{Cl}$, confidence interval; $N$, number; PCN, percutaneous nephrostomy insertion.

blood loss, total number of surgical re-interventions within 12 months (including nonurological re-interventions), UTIs, urosepsis, deaths, wound infections, and readmissions within 1 and 12 months after transplantation. In the stent group, there were more patients with macroscopic hematuria than in the no-stent group, $26 \%$ (95\% CI 13.1-38.9\%). In addition, compared to the nostent group, more patients in the stent group required treatment because of graft rejection within 1 month after transplantation, $15 \% \quad(95 \%$ CI $2.7-27.3 \%)$ (Table 3). Stented patients had a longer hospital stay because of our internal protocol (discharge only after stent removal) (mean: $13 \pm 6$ vs. $10 \pm 4$ days). In the no-stent group, one patient died of non-Hodgkin lymphoma. In the stent group, two patient died: one because of respiratory insufficiency caused by GuillainBarre syndrome and the other patient because of cardiac reasons.

\section{Graft outcome}

We did not detect any differences between both groups regarding the mean GFR on days 7 or 14 or at $1,3,6$, or 12 months after transplantation (Table 4).

\section{Quality of life questionnaires}

We compared QOL outcomes correcting for baseline at the various time points using a linear mixed model. Besides a better Euro-Qol-5D in the no-stent group 2 and 6 weeks postoperative, we could not demonstrate an effect of the stent $(P=0.56$ for the multivariable test for the SF-36 and $P=0.06$ for the multivariable test for the Euro-Qol-5D) (Tables 5 and 6).

\section{Discussion}

This randomized controlled trial in living-donor kidney transplantation investigated the influence of stent versus no-stent placement. We found more PCN insertions, urinary leakages, and surgical re-interventions because of urological complications in the no-stent group. In the stent group, there were more hematuria and graft rejection. In general, we could not demonstrate an effect of the stent on quality of life, besides a better EuroQol-5D result in the no-stent group 2 and 6 weeks postoperative.

This trial was conducted to provide well-defined, evidence-based arguments for ureteric stent placement in kidney transplantation. Previously, five randomized controlled trials [15-19] were conducted on stent placement, but they differed in the use of living-donor or deceased-donor kidneys, intravesical or extravesical anastomoses, and the type of stent used. According to those studies, stenting seemed to be favored, but it remained uncertain whether stenting should be considered routine or only performed when strictly defined criteria were met. A Cochrane review on this topic supported the use of ureteral stents, but they did not state recommendations for duration and the type of stent [4]. Timing of stent removal remains difficult. A recently published meta-analysis supports stent removal within three weeks postoperatively; however, this statement was based on double J stents [20].

Previously, we investigated whether the type of anastomosis (extravesical vs. intravesical; INEX trial) was a risk factor for PCN insertion [21]. Both groups received a ureteral stent, although a different stent than which was used in the current SPLINT trial. We found no difference in the number of PCN insertions between groups that received intravesical or extravesical anastomoses $(20 \%$ vs. $20 \%)$. However, the number of UTIs was lower in the extravesical group. Currently, our standard of care includes the extravesical anastomosis.

Based on the results of the current study, we are convinced that ureteric stent placement with an extravesical ureteroneocystostomy could reduce the number of urological complications in kidney transplantation. Only 


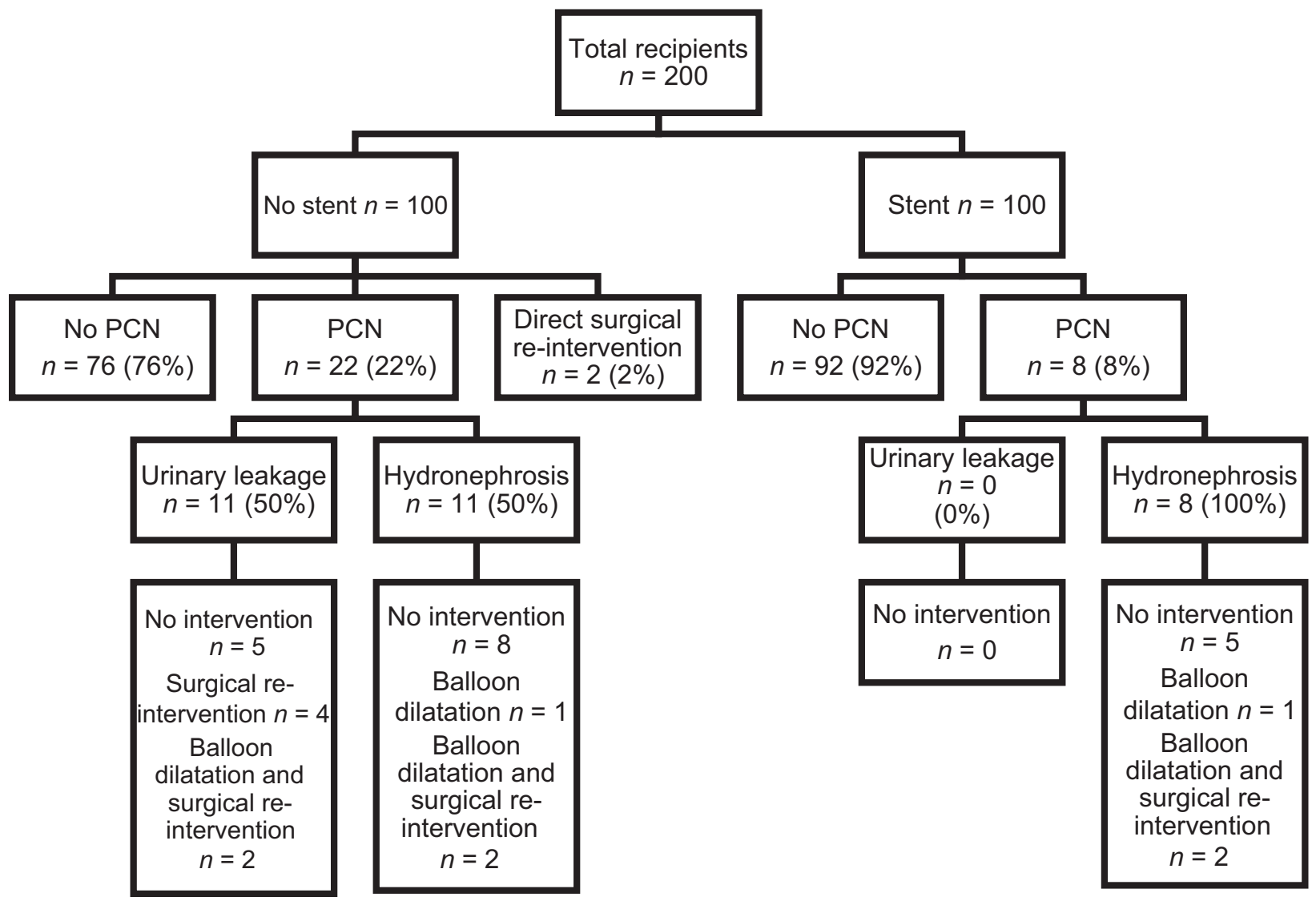

Figure 1 Flowchart SPLINT trial. N, number; PCN, percutaneous nephrostomy.

Table 3. Overall outcome

\begin{tabular}{|c|c|c|c|c|}
\hline Outcome & Total $(N=200)$ & No stent $(N=100)$ & Stent $(N=100)$ & $P$-value \\
\hline Duration of surgery, min; mean $\pm S D$ & $116 \pm 36$ & $114 \pm 39$ & $119 \pm 34$ & 0.314 \\
\hline Blood loss, ml; median (range) & $150(0-2000)$ & $150(0-2000)$ & $150(0-1300)$ & 0.451 \\
\hline Any surgical re-intervention in $<12$ months, $N(\%)$ & $34(17)$ & $21(21)$ & $13(13)$ & 0.132 \\
\hline Hematuria in <1 month, $N(\%)$ & $126(63)$ & $50(50)$ & $76(76)$ & $<0.001$ \\
\hline UTI in $<1$ month, $N(\%)$ & $47(24)$ & $27(27)$ & $20(20)$ & 0.243 \\
\hline Urosepsis in $<1$ month, $N(\%)$ & $9(5)$ & $5(5)$ & $4(4)$ & 0.733 \\
\hline Wound infection in $<1$ month, $N(\%)$ & $22(11)$ & $14(14)$ & $8(8)$ & 0.175 \\
\hline Rejection in $<1$ month, $N(\%)$ & $57(29)$ & $21(21)$ & $36(36)$ & 0.019 \\
\hline Biopsy-proven rejection in $<1$ month, $N(\%)$ & $40(20)$ & $11(11)$ & $29(29)$ & 0.001 \\
\hline Total length of hospital stay, days; mean \pm SD & $11 \pm 5$ & $10 \pm 4$ & $13 \pm 6$ & $<0.001$ \\
\hline Readmission in $<1$ month, $N(\%)$ & $47(24)$ & $27(27)$ & $20(20)$ & 0.243 \\
\hline Readmission in $<12$ months, $N(\%)$ & $110(55)$ & $57(57)$ & $53(53)$ & 0.570 \\
\hline $\begin{array}{l}\text { Number of readmissions per person } \\
\text { in }<12 \text { months; median (range) }\end{array}$ & $1(0-17)$ & $1(0-12)$ & $1(0-17)$ & 0.563 \\
\hline Death $<12$ months, $N(\%)$ & $3(1.5)$ & $1(1)$ & $2(2)$ & 0.561 \\
\hline
\end{tabular}

$N$, number; SD, standard deviation; UTI, urinary tract infection.

Bold values are statistically significant.

$8 \%$ of patients received PCN insertions in the stented extravesical anastomosis group. This proportion was considerably lower than the $20 \%$ in the above-mentioned INEX trial [21].
Although stent placement increased the duration of ureteral anastomosis, it did not influence the total duration of surgery. However, the mean total hospital stay was longer in the stent group than in the no-stent 
Table 4. Graft outcome

\begin{tabular}{llll}
\hline $\begin{array}{l}\text { Renal function } \\
\text { after KT } \\
\text { mean } \pm \text { SD }\end{array}$ & $\begin{array}{l}\text { No stent } \\
(N=100)\end{array}$ & $\begin{array}{l}\text { Stent } \\
(N=100)\end{array}$ & \\
\hline 7 days & $41 \pm 15$ & $41 \pm 19$ & 0.950 \\
14 days & $46 \pm 15$ & $46 \pm 17$ & 0.778 \\
1 month & $48 \pm 15$ & $47 \pm 16$ & 0.794 \\
3 months & $49 \pm 15$ & $48 \pm 16$ & 0.576 \\
6 months & $51 \pm 16$ & $49 \pm 16$ & 0.324 \\
12 months & $52 \pm 18$ & $52 \pm 18$ & 0.922 \\
\hline
\end{tabular}

GFR, glomerular filtration rate; KT, kidney transplantation; SD, standard deviation.

group. This is due to the fact that in our hospital, stented patients were only discharged after stent removal.

Remarkably, we found a higher number of rejections in the stent group than in the no-stent group. These patients had received antirejection treatments (intravenous methylprednisolone, IVIG, alemtuzumab, or rATG) within 1 month after transplantation. We could not find any explanation for this finding. We speculate that, because patients with stents had prolonged hospital stays, rise of serum creatinine might have been detected more rapidly, because of frequent in-hospital evaluations, compared with outpatient visits. In addition, urine production can be monitored more accurately in patients with externalized stents than in patients without a stent; this monitoring might have led to a relatively low threshold for biopsy. However, we cannot completely disclaim that the stent (being a foreign body) could facilitate an immune response leading to rejection. Note that there is no literature, which does substantiate this.

This study had a few limitations. Most importantly, the suprapubic externalized type of stent (single J stent) used in this trial is not a stent commonly used. Most transplant centers use the double J stent, and literature indicated that the double $\mathrm{J}$ stent is also associated with minor urological complications. Furthermore, we have a relatively high number of PCN insertions compared to literature. In our clinic, we have a low threshold to place a PCN, as this is considered a minimally invasive event. Even a mild hydronephrosis leads to PCN insertion, either for therapeutically benefit or as diagnostic tool before performing an biopsy. As in this trial the data are collected prospectively, the database is more complete and accurate, possibly resulting in a higher percentage of complications than the complication rates that are mentioned in other kinds of publications. Our

Table 5. SF-36 questionnaire

\begin{tabular}{|c|c|c|c|c|c|c|c|c|}
\hline \multirow[b]{2}{*}{ Time point } & \multirow{2}{*}{$\begin{array}{l}\text { No stent } \\
\text { Mean }\end{array}$} & \multicolumn{2}{|l|}{$95 \% \mathrm{Cl}$} & \multirow{2}{*}{$\begin{array}{l}\text { Stent } \\
\text { Mean }\end{array}$} & \multicolumn{2}{|l|}{$95 \% \mathrm{Cl}$} & \multirow{2}{*}{$\begin{array}{l}\text { Delta } \\
\text { Mean }\end{array}$} & \multirow[b]{2}{*}{$P$-value } \\
\hline & & Lower limit & Upper limit & & Lower limit & Upper limit & & \\
\hline 2 weeks & 21.2 & 13.3 & 29.2 & 18.1 & 10.3 & 26.0 & -3.1 & 0.272 \\
\hline 6 weeks & 30.3 & 22.3 & 38.3 & 26.9 & 18.9 & 34.9 & -3.5 & 0.241 \\
\hline 3 months & 32.9 & 24.7 & 41.1 & 35.7 & 27.6 & 43.8 & 2.8 & 0.381 \\
\hline 6 months & 35.8 & 27.7 & 44.0 & 34.7 & 26.6 & 42.8 & -1.1 & 0.720 \\
\hline 9 months & 40.2 & 31.9 & 48.5 & 38.7 & 30.5 & 47.0 & -1.4 & 0.666 \\
\hline 12 months & 40.8 & 32.6 & 49.0 & 39.3 & 31.2 & 47.4 & -1.5 & 0.639 \\
\hline Preoperative scores & 59.4 & 55.3 & 63.4 & 57.9 & 53.9 & 62.0 & -1.5 & \\
\hline
\end{tabular}

Table 6. Euro-Qol-5D questionnaire

\begin{tabular}{|c|c|c|c|c|c|c|c|c|}
\hline \multirow[b]{2}{*}{ Postoperative time point } & \multirow{2}{*}{$\begin{array}{l}\text { No stent } \\
\text { Mean }\end{array}$} & \multicolumn{2}{|l|}{$95 \% \mathrm{Cl}$} & \multirow{2}{*}{$\begin{array}{l}\text { Stent } \\
\text { Mean }\end{array}$} & \multicolumn{2}{|l|}{$95 \% \mathrm{Cl}$} & \multirow{2}{*}{$\begin{array}{l}\text { Delta } \\
\text { Mean }\end{array}$} & \multirow[b]{2}{*}{$P$-value } \\
\hline & & Lower limit & Upper limit & & Lower limit & Upper limit & & \\
\hline 2 week & -0.17 & -0.22 & -0.12 & -0.24 & -0.30 & -0.19 & -0.07 & 0.030 \\
\hline 6 weeks & -0.07 & -0.12 & -0.02 & -0.14 & -0.20 & -0.09 & -0.07 & 0.037 \\
\hline 3 months & -0.07 & -0.12 & -0.02 & -0.07 & -0.13 & -0.02 & 0.00 & 0.917 \\
\hline 6 months & -0.06 & -0.11 & -0.01 & -0.10 & -0.15 & -0.04 & -0.04 & 0.230 \\
\hline 9 months & -0.07 & -0.12 & -0.02 & -0.05 & -0.11 & 0.00 & 0.02 & 0.628 \\
\hline 12 months & -0.08 & -0.13 & -0.03 & -0.08 & -0.14 & -0.02 & 0.00 & 0.984 \\
\hline Preoperative scores & -0.17 & -0.21 & 0.12 & -0.21 & -0.25 & 0.16 & -0.04 & \\
\hline
\end{tabular}

Bold values are statistically significant. 
number of re-interventions because of urological complications is comparable to literature. Cost-effectiveness data were collected during this trial and will be published separately.

A previous retrospective study by Vogel et al. that included 76 patients compared 43 externalized stents and 33 double $J$ stents. They reported that the incidences of leakage from the ureteroneocystostomy were $13.9 \%$ for externalized stents and $0 \%$ for double $\mathrm{J}$ stents. Furthermore, they found a 2-day reduction in hospital stay time with the internal stent [22]. Gomes et al. also retrospectively reviewed the use of externalized stents, internal stents, and no stent, in 2061 recipients of kidney transplants. In their cohort, the incidences of urological complications were $17.3 \%$ in the externalized stent group, $8.4 \%$ in the no-stent group, and $5.4 \%$ in the double $J$ stent group $(P<0.0005)$ [23]. The authors concluded that externalized stents should be avoided, because they were associated with a high urological complication rate. Guleria et al. [24] also reduced urological complications by changing their technique from no stent $(7.7 \%)$ to a double J stented (for a period of 6 weeks) ureteroneocystostomy (3.8\%). Unfortunately, those studies had retrospective designs. Furthermore, patients with an external stent do not need an additional cystoscopy for stent removal. By lowering the number of interventions, external stents may reduce patients' morbidity and costs. Therefore, we recently started a new trial at the
Erasmus MC, University Medical Center, Rotterdam, in which we investigate whether single J or double J stenting is superior in reducing the number of urological complications.

In this trial, noninferiority has not been demonstrated for no-stent placement in relation to the number of urological complications.

\section{Authorship}

L.S.S.O.: performed research, analyzed data and wrote the manuscript. R.C.M., F.J.M.F.D., H.J.A.N.K., T.C.K.T., H.H. and J.v.d.W.: performed research and wrote the manuscript. S.P.W.: analyzed data. J.N.M.I. and T.T.: designed research, performed research, analyzed data and wrote the manuscript.

\section{Funding}

This study was supported by Stichting Coolsingel.

\section{Conflict of interest}

The authors have declared no conflicts of interest.

\section{Acknowledgments}

The authors wish to thank database managers Carola Zandijk and Anneke van Duuren-van Pelt.

\section{REFERENCES}

1. Dols LF, Terkivatan T, Kok NF, et al. Use of stenting in living donor kidney transplantation: does it reduce vesicoureteral complications? Transplant Proc 2011; 43: 1623.

2. Miraglia R, Caruso S, Milazzo M, Salis P, Luca A, Gridelli B. Efficacy of interventional radiology procedures for the treatment of early ureteral complications after kidney transplantation. Transplant Proc 2006; 38: 2919.

3. Wilson CH, Rix DA, Manas DM. Routine intraoperative ureteric stenting for kidney transplant recipients. Cochrane Database Syst Rev 2013; 6: CD004925.

4. Wilson $\mathrm{CH}$, Bhatti AA, Rix DA, Manas DM. Routine intraoperative ureteric stenting for kidney transplant recipients. Cochrane Database Syst Rev 2005; 4: CD004925.
5. Sarier M, Demir M, Duman I, Yuksel Y, Demirbas A. Evaluation of ureteral stent colonization in live-donor renal transplant recipients. Transplant Proc 2017; 49: 415.

6. Veltman Y, Shields JM, Ciancio G, Bird VG. Percutaneous nephrolithotomy and cystolithalapaxy for a "forgotten" stent in a transplant kidney: case report and literature review. Clin Transplant 2010; 24: 112.

7. Kumar A, Verma BS, Srivastava A, Bhandari M, Gupta A, Sharma R. Evaluation of the urological complications of living related renal transplantation at a single center during the last 10 years: impact of the Double-J* stent. J Urol 2000; 164: 657.

8. Rigg KM, Proud G, Taylor RM. Urological complications following renal transplantation. A study of 1016 consecutive transplants from a single centre. Transpl Int. 1994; 7: 120.

9. Mangus RS, Haag BW. Stented versus nonstented extravesical ureteroneocystostomy in renal transplantation: a metaanalysis. $A m \mathrm{~J}$ Transplant 2004; 4: 1889.

10. Gregoir W. Le Traitement Chirurgical $\mathrm{Du}$ Reflux V'esico-Ur'et'eral Cong'enital [the Surgical Treatment of Congenital Vesico-Ureteral Reflux]. Acta Chir Belg 1964; 63: 431.

11. Lich R Jr, Howerton LW, Davis LA. Childhood urosepsis. J Ky Med Assoc 1961; 59: 1177.

12. Rabin R, Gudex C, Selai C, Herdman M. From translation to version management: a history and review of methods for the cultural adaptation of the EuroQol five-dimensional questionnaire. Value Health 2014; 17: 70. 
13. Ware JE Jr, Sherbourne CD. The MOS 36-item short-form health survey (SF36). I. Conceptual framework and item selection. Med Care 1992; 30: 473.

14. Shan G, Wang W. ExactCIdiff: an R package for computing exact confidence intervals for the difference of two proportions. $R J$ 2013; 5: 62 .

15. Bassiri A, Amiransari B, Yazdani M, Sesavar Y, Gol S. Renal transplantation using ureteral stents. Transplant Proc 1995; 27: 2593.

16. Benoit G, Blanchet P, Eschwege P, Alexandre L, Bensadoun $\mathrm{H}$, Charpentier B. Insertion of a double pigtail ureteral stent for the prevention of urological complications in renal transplantation: a prospective randomized study. J Urol 1996; 156: 881.

17. Dominguez J, Clase CM, Mahalati K, et al. Is routine ureteric stenting needed in kidney transplantation? A randomized trial. Transplantation 2000; 70: 597.

18. Kumar A, Kumar R, Bhandari M. Significance of routine JJ stenting in living related renal transplantation: a prospective randomised study. Transplant Proc 1998; 30: 2995.

19. Pleass HC, Clark KR, Rigg KM, et al. Urologic complications after renal transplantation: a prospective randomized trial comparing different techniques of ureteric anastomosis and the use of prophylactic ureteric stents. Transplant Proc 1995; 27: 1091.

20. Visser IJ, van der Staaij JPT, Muthusamy A, Willicombe M, Lafranca JA, Dor F. Timing of ureteric stent removal and occurrence of urological complications after kidney transplantation: a systematic review and meta-analysis. J Clin Med 2019; 8: 689.
21. Slagt IK, Dor FJ, Tran TC, et al. A randomized controlled trial comparing intravesical to extravesical ureteroneocystostomy in living donor kidney transplantation recipients. Kidney Int 2014; 85: 471.

22. Vogel T, Utech $M$, Schmidt F, et al. Double-J versus external ureteral stents in kidney transplantation: a retrospective analysis. Nephrourol Mon 2015; 7: e27820.

23. Gomes G, Nunes P, Castelo D, et al. Ureteric stent in renal transplantation. Transplant Proc 2013; 45: 1099.

24. Guleria S, Chahal R, Madaan S, et al. Ureteric complications of renal transplantation: the impact of the double J stent and the anterior extravesical ureteroneocystostomy. Transplant Proc 2005; 37: 1054. 